\title{
Potential novel antibiotics from HTS targeting the virulence-regulating transcription factor, VirF, from Shigella flexneri
}

\begin{abstract}
Anthony A Emanuele ${ }^{1}$, Nancy E Adams ${ }^{2}$, Yi-Chen Chen $^{1}$, Anthony T Maurelli ${ }^{2}$ and George A Garcia ${ }^{1}$
VirF is an AraC-type transcriptional regulator responsible for activating the transcription of virulence genes required for the intracellular invasion and cell-to-cell spread of Shigella flexneri. Gene disruption studies have validated VirF as a potential target for an anti-virulence therapy to treat shigellosis by determining that VirF is necessary for virulence, but not required for bacterial viability. Using a bacteria-based, $\beta$-galactosidase reporter assay we completed a high-throughput screening (HTS) campaign monitoring VirF activity in the presence of over 140000 small molecules. From our screening campaign, we identified five lead compounds to pursue in tissue culture-based invasion and cell-to-cell spread assays, and toxicity screens. Our observations of activity in these models for infection have validated our approach of targeting virulence regulation and have allowed us to identify a promising chemical scaffold from our HTS for hit-to-lead development. Interestingly, differential effects on invasion versus cell-to-cell spread suggest that the compounds' efficacies may depend, in part, on the specific promoter that VirF is recognizing.
\end{abstract}

The Journal of Antibiotics (2014) 67, 379-386; doi:10.1038/ja.2014.10; published online 19 February 2014

Keywords: drug resistance; pathogenicity; type III secretion system

\section{INTRODUCTION}

The global rise of multidrug-resistant pathogens is a serious challenge to human health. The emergence and spread of resistance to drugs considered to be last resort, such as the plasmid-encoded New Delhi metallo- $\beta$-lactamase that confers resistance to all $\beta$-lactams, even carbapenems, ${ }^{1}$ is a major concern to health officials worldwide. Exacerbating this problem is the slow rate at which new antibiotics are being discovered. Since the 1960s, no new class of broad-spectrum antibiotics has been discovered and only four classes of narrowspectrum antibiotics (streptogramins: streptogramin B, oxazolidinones: linezolid, lipopeptides: daptomycin and diarylquinolines: bedaquiline) have had compounds approved for clinical use. ${ }^{2}$ Unfortunately, resistance to all four newly approved compounds has already been observed. ${ }^{3-6}$

All current antibiotics negatively affect bacterial viability, and as such, any organisms that mutate to become resistant to the antibiotic will have a selective growth advantage. This can (or often) result in the rapid emergence of resistant strains. A potential method to combat bacterial infection while minimizing the emergence of resistance is to target virulence instead of bacterial viability. It is hypothesized that targeting virulence will result in less selective pressure for the emergence of new drug-resistant strains as they are expected to have little or no growth advantage. ${ }^{7-9}$ Also, virulence therapies should not harm the normal microbiota of the host, as the therapy targets virulence pathways that do not exist in the nonpathogenic microbiota. Although virulence therapies sound promising, they are still in the early phases of development; however, recent studies have provided encouraging evidence that this strategy could be effective. Virstatin, a small molecule inhibitor of ToxT (a transcriptional activator of critical virulence factors in Vibrio cholerae) was shown to protect infant mice from intestinal colonization by V. cholerae. ${ }^{10}$ Also, a series of benzimidazole compounds were shown to limit infection in a murine model of Yersinia pseudotuberculosis pneumonia through the inhibition of the transcriptional regulator of the Yersinia type III secretion system, LcrF.9,11,12

We have previously conducted initial studies to discover an inhibitor of $\operatorname{VirF}^{13}$ (Emanuele et al., unpublished), the main transcriptional activator of virulence genes in S. flexneri. We believe that VirF is an ideal target for an anti-virulence therapy for shigellosis as gene disruption studies have shown that it is required for host infection, but not bacterial viability. ${ }^{14}$ As shown in Figure 1, VirF controls three essential processes that are necessary for host cell infection. VirF activates the transcription of virulence factors that are required for macrophage apoptosis and escape, intestinal epithelial cell invasion and cell-to-cell spread. We hypothesize that attenuation of VirF activity at any of these steps would reduce virulence.

${ }^{1}$ Department of Medicinal Chemistry, College of Pharmacy, University of Michigan, Ann Arbor, MI, USA and ${ }^{2}$ Department of Microbiology and Immunology, Uniformed Services University of the Health Sciences, Bethesda, MD, USA

Correspondence: Professor GA Garcia, Department of Medicinal Chemistry, College of Pharmacy, University of Michigan, 428 Church Street, Ann Arbor, MI 48109-1065, USA. E-mail: gagarcia@umich.edu

Received 14 October 2013; revised 17 December 2013; accepted 14 January 2014; published online 19 February 2014 


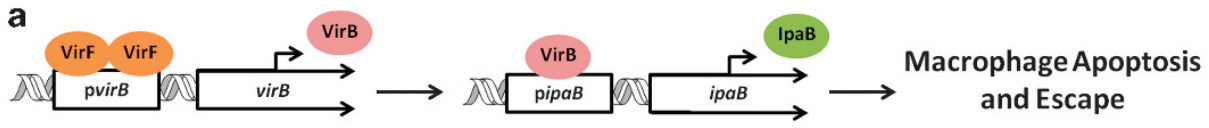

b

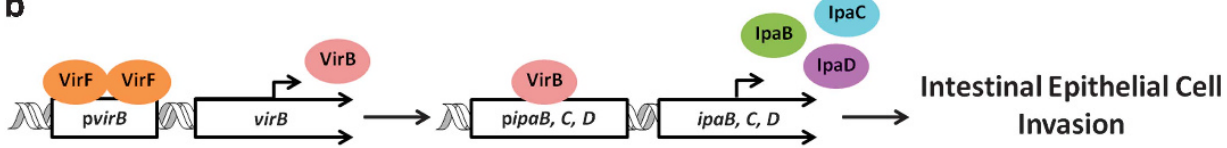

C

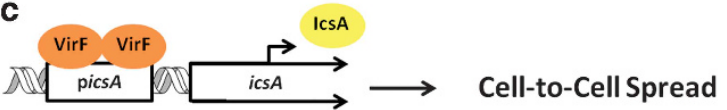

Figure 1 Processes essential for host cell infection activated by VirF. (a) Macrophage apoptosis and escape: VirF binds to the virB promoter and activates the transcription of the secondary transcriptional regulator, VirB. ${ }^{27}$ VirB activates the transcription of IpaB. ${ }^{28}$ Once inside the macrophage, IpaB binds to caspase-1, which induces macrophage apoptosis allowing the Shigella to escape the macrophage. ${ }^{29}$ (b) Intestinal epithelial cell invasion: The secondary transcriptional regulator, VirB, also activates the transcription of IpaC and IpaD. ${ }^{27,28}$ Together with IpaB, IpaC and IpaD form the tip of the type-three secretion system (T3SS) needle that inserts into the basolateral side of host intestinal epithelial cells. ${ }^{30,31}$ The T3SS releases effector proteins into the host cell that promote bacterial invasion. ${ }^{32}$ (c) Cell-to-cell spread: VirF binds to the icsA promoter and activates the transcription of IcsA. ${ }^{33}$ IcsA is part of the complex that polymerizes host cell actin allowing the Shigella to become motile and spread from cell to cell. ${ }^{34}$

In our previous studies, we used a bacteria-based, VirF-driven, $\beta$-galactosidase reporter assay to screen $\sim 42000$ small molecules to identify inhibitors of VirF. ${ }^{13}$ The reporter assay monitored the activation of the virB promoter by VirF. In currently unpublished work, we have also screened 20000 natural product extracts using the same reporter assay (Emanuele et al., unpublished). From the two high-throughput screens we were able to identify seven small molecules and 24 natural product extracts that inhibited the activity of VirF. While challenging to follow-up on, the natural product extract screen has already identified one known antibiotic and more extracts remain to be further characterized (Emanuele et al., unpublished). The library for our previous small molecule screen was modest in size $(\sim 42000)$. In order to increase the range of chemical diversity to which our screens were exposed, we report here the extension of our small molecule screen to include $\sim 100000$ more compounds, for a total small molecule screen of $\sim 140000$ compounds. Through a series of counter and confirmation screens we were able to narrow our hits down to five lead compounds. These lead compounds were then tested in tissue culture-based invasion and plaque formation assays to determine their ability to prevent initial host cell infection and spread. Our observations of activity in these models for infection have both validated our approach of targeting virulence regulation and have allowed us to identify a promising chemical scaffold from our HTS for hit-to-lead development of an anti-virulence therapeutic for the treatment of shigellosis.

\section{MATERIALS AND METHODS}

\section{Reagents}

All reagents were purchased from Sigma-Aldrich (St Louis, MO, USA), unless otherwise specified. Chlorophenol red $\beta$-D-galactopyranoside (CPRG) was purchased from Roche (Basel, Switzerland). Yeast extract, bactotryptone, carbenicillin and Corning microtiter plates (384 and 96 wells) were purchased from Fisher Scientific (Hampton, NH, USA). Compounds selected for reconfirmation screening were purchased from ChemDiv (seven compounds, San Diego, CA, USA) and Vitas-M Laboratory (three compounds, Moscow, Russia).

\section{Strains and plasmids}

Wild-type S. flexneri serotype 2a strain, 2457T, ${ }^{15}$ and an isogenic, virulence plasmid-cured derivative, BS103, ${ }^{16}$ were used in this study. HeLa cells and L2 mouse fibroblasts were used for virulence assays and were grown in
Dulbecco's modified Eagle medium (DMEM; LifeTechnologies; Grand Island, NY, USA), supplemented with $10 \%$ heat-inactivated fetal bovine serum (LifeTechnologies). The bacteria were grown in tryptic soy broth (TSB) or on agar plates supplemented with $0.025 \%$ Congo Red agar as necessary at $37^{\circ} \mathrm{C}$. The construction of the reporter plasmids, pMALvirF-lac $Z$ and $\mathrm{p} M A L(\Delta v i r F)$-lac $Z$ (positive control), has been previously described. ${ }^{13}$

\section{Small molecule library}

A 100000 compound library produced by ChemDiv was screened at the Center for Chemical Genomics (University of Michigan, Ann Arbor).

\section{High-throughput VirF-driven, $\beta$-galactosidase reporter assay}

The construction and optimization of the reporter assay has been previously described. ${ }^{13}$ In brief, starter cultures of $S$. flexneri BS103 harboring either the reporter plasmid ( $\mathrm{pMALvirF-lacZ)} \mathrm{or} \mathrm{positive} \mathrm{control} \mathrm{plasmid} \mathrm{(} \mathrm{p} M A L(\Delta v i r F)$ lac $Z$ ) were grown overnight at $37^{\circ} \mathrm{C}$ with shaking in $2 x T Y$ media $(16 \mathrm{~g}$ bactotryptone, $10 \mathrm{~g}$ yeast extract, $5 \mathrm{~g} \mathrm{NaCl}$ per liter of water supplemented with $100 \mu \mathrm{g} \mathrm{ml}^{-1}$ carbenicillin). The next day, $20 \mu \mathrm{l}$ of $2 \mathrm{xTY}$ media supplemented with $100 \mu \mathrm{g} \mathrm{ml}^{-1}$ carbenicillin was added to 384-well plates using a multidrop dispenser (Thermo Scientific, Waltham, MA, USA). Compounds from the $100-\mathrm{K}$ ChemDiv library $(13 \mu \mathrm{M}$ final, $n=2,0.2 \mu \mathrm{l})$ were then added to appropriate wells using the Biomek HDR pintool instrument (Beckman, Fullerton, CA, USA). Overnight starter cultures (reporter plasmid and positive control) were diluted to $\mathrm{OD}_{600}=0.012$ using $2 \mathrm{xTY}$ media supplemented with $100 \mu \mathrm{g} \mathrm{ml}^{-1}$ carbenicillin. The diluted cultures $(10 \mu \mathrm{l})$ were added to the appropriate wells of the plates via the multidrop dispenser (total volume $30 \mu \mathrm{l}$ ). Plates were then spun-down at $1000 \mathrm{~g}$ for $1 \mathrm{~min}$ using a Beckman Coulter Allegra Series centrifuge. After centrifugation, plates were placed overnight $(\sim 20 \mathrm{~h})$ in a humidified, $30^{\circ} \mathrm{C}$ incubator (VWR, Radnor, PA, USA). The following day, $30 \mu \mathrm{l}$ of CPRG solution $\left(0.5 \mathrm{mg} \mathrm{ml}^{-1}\right.$ CPRG, $0.1 \%$ Triton X-100, $60 \mathrm{~mm} \mathrm{Na}_{2} \mathrm{HPO}_{4}, 40 \mathrm{~mm} \mathrm{NaH} \mathrm{PO}_{4}, 10 \mathrm{~mm} \mathrm{KCl}, 1 \mathrm{~mm} \mathrm{MgSO}_{4}, \mathrm{pH} 7.0$ ) was added to each well. Plates were incubated at room temperature for $10 \mathrm{~min}$ before measuring $\mathrm{CPR}$ absorbance $\left(\mathrm{A}_{570}\right)$ in a PHERAstar (BMG Labtech, Cary, NC, USA) plate reader with a narrow bandpass filter.

\section{Counter screens}

Potential hits identified from the primary high-throughput screen were subjected to a series of stringent control screens. First, compounds were assayed in a dose-response study $(n=2)$ following the screening protocol described above. However, for the dose-response study, the concentration of the compounds was varied using twofold serial dilutions ranging from 100 to $0.78 \mu \mathrm{m}$. Also, directly before the addition of the CPRG solution, bacteria density $\left(\mathrm{OD}_{600}\right)$ was measured using the PHERAstar plate reader to determine whether the compounds inhibited bacterial growth. 
Compounds that inhibited VirF in a dose-dependent manner, but did not inhibit bacterial growth (for example, $\mathrm{MIC}_{50}>100 \mu \mathrm{M}$ ) were selected for $\beta$-galactosidase inhibition screening. For the $\beta$-galactosidase inhibition screening, cultures of S. flexneri BS103 harboring either pMALvirF-lacZ or $\mathrm{p} M A L(\Delta v i r F)-$ lac $Z$ were diluted to $\mathrm{OD}_{600}=1.0$ using $2 \mathrm{xTY}$ media supplemented with $100 \mu \mathrm{g} \mathrm{ml}^{-1}$ carbenicillin and added to appropriate wells of a 384-well microtiter plate using the multidrop dispenser $(30 \mu \mathrm{l})$. Compounds were added to the plates in duplicate, and their concentrations were varied using twofold serial dilutions ranging from 100 to $0.78 \mu \mathrm{M}$. Immediately following compound addition, CPRG solution was added to each well $(30 \mu \mathrm{l})$. After a 10-min incubation period at room temperature, CPR absorbance $\left(\mathrm{A}_{570}\right)$ was measured using the PHERAstar plate reader to determine whether the compounds directly inhibited $\beta$-galactosidase.

\section{Reconfirmation screen}

Fresh, neat samples of compounds identified as hits were ordered from commercial vendors for a reconfirmation dose-response study. For this study, the screening protocol was modified to a 96-well microtiter plate format. Working stocks of each compound were made using 2xTY media supplemented with $100 \mu \mathrm{g} \mathrm{ml}^{-1}$ carbenicillin. Working stocks were added to the plates in triplicate using a twofold serial dilution technique $(60 \mu \mathrm{l})$. Cultures of S. flexneri BS103 harboring either pMALvirF-lacZ or pMAL( $\Delta$ virF)-lacZ were diluted to $\mathrm{OD}_{600}=0.012$ using 2 XTY media supplemented with $100 \mu \mathrm{g} \mathrm{ml}^{-1}$ carbenicillin and added to appropriate wells of plate $(30 \mu \mathrm{l})$. Final compound concentrations ranged from either 100 to $0.78 \mu \mathrm{M}$ or 50 to $0.78 \mu \mathrm{M}$. For negative controls, compound vehicle (dimethyl sulfoxide, $1 \%$ final concentration) was added to wells instead of compound. Plates were placed overnight $(\sim 20 \mathrm{~h})$ in a humidified, $30^{\circ} \mathrm{C}$ incubator. The following day, bacteria density $\left(\mathrm{OD}_{600}\right)$ was measured using a Biotek Synergy H1 plate reader (Winooski, VT, USA), and then $90 \mu \mathrm{l}$ of CPRG solution was added to each well. Plates were allowed to incubate for $7 \mathrm{~min}$ at room temperature, and then CPR absorbance $\left(\mathrm{A}_{570}\right)$ was read using the Biotek plate reader.

\section{Data analysis/hit selection}

Three different sets of selection criteria were used to define active compounds in the initial high-throughput screen. Samples were defined as active if either (A) one of their $A_{570}$ readings was more than or equal to three times the s.d. of the negative controls away from the average $A_{570}$ of the negative controls (calculated on a plate-by-plate basis); or (B) one of their $\mathrm{A}_{570}$ readings was more than or equal to three times the s.d. of the plate (not including positive controls) away from the average $A_{570}$ of the plate (not including positive controls, calculated on a plate-by-plate basis); or (C) if their percent effect value (calculated on a plate-by-plate basis using both negative and positive controls) was $\geqslant 30 \%$. Compounds that passed initial selection criteria were tested in a series of counter screens. Compounds that passed all counter screens were classified as hits. Counter screen selection criteria were as follows: $\mathrm{IC}_{50} \leqslant 100 \mu \mathrm{M}$ for $\mathrm{VirF}$ inhibition dose-response study, $\mathrm{MIC}_{50}>100 \mu \mathrm{M}$ for bacterial growth inhibition and no direct $\beta$-galactosidase inhibition over the range of concentrations tested. For all dose-response studies, data were fit by nonlinear regression to following equation using Kaleidagraph (Synergy Software, Essex, VT, USA):

$$
y=\text { lower }+\left[(\text { upper }- \text { lower }) /\left(1+10^{(\mathrm{M} 0-\mathrm{M} 1) \times \mathrm{M} 2}\right)\right]
$$

where $\mathrm{M} 0=\log$ of compound concentration, $\mathrm{M} 1=\log$ of $\mathrm{IC}_{50}, \mathrm{M} 2=$ hill slope, 'lower' is defined to be the lower limit of the assay (lower $=0$ ), and 'upper' is defined to be the upper limit of the assay (upper $=100$ ).

\section{Growth curves}

Cultures of bacteria were grown overnight in TSB, washed in phosphatebuffered saline solution (PBS; Lonza; Walkersville, MD, USA) and diluted in TSB with appropriate concentration of drug. Approximately $2 \times 10^{4}$ colony forming units were applied to individual wells in a 96-well plate for growth curve analysis. Growth curves were performed in a BioTek Synergy2 plate reader at $37^{\circ} \mathrm{C}$ with constant shaking. Readings of OD at $600 \mathrm{~nm}$ were recorded every $30 \mathrm{~min}$ for $24 \mathrm{~h}$, and data points were analyzed with Gen5 version 1.11.5 software (Winooski, VT, USA).

\section{Cell toxicity}

Serial dilutions of each compound were applied to 50\% confluent monolayers of HeLa and L2 cells and monitored daily, up to 3 days, for growth phenotypes. Physical signs of cell health were monitored as an OD reading could not be determined with adherent cells (Supplementary Table 2). The highest tolerable compound concentration with HeLa cells was then used to assess bacterial growth of wild-type S. flexneri 2457T, as well as a virulence plasmid-cured strain (BS103), by measuring $\mathrm{OD}_{600}$ for $24 \mathrm{~h}$.

\section{Invasion assay}

Assays were performed as previously described. ${ }^{17}$ In brief, bacteria were grown overnight, subcultured into TSB supplemented with compound when appropriate and grown with agitation until reaching mid-log phase. Strains were standardized to an OD at $600 \mathrm{~nm}$ of 0.35 , washed in PBS and resuspended in DMEM (Gibco; Grand Island, NY, USA) supplemented with compound when appropriate. The input bacteria were titered on TSB Congo Red plates before applying to six-well plates seeded to semiconfluence with HeLa cells. The plates were then centrifuged at 3000 r.p.m. for $10 \mathrm{~min}$ and then permitted to invade for $30 \mathrm{~min}$ at $37^{\circ} \mathrm{C}$ with $5 \% \mathrm{CO}_{2}$. Following a wash with PBS, the monolayers were incubated at $37^{\circ} \mathrm{C}$ with $5 \% \mathrm{CO}_{2}$ for $30 \mathrm{~min}$ in DMEM supplemented with compound (when necessary) and $50 \mu \mathrm{gl}^{-1}$ gentamicin. After washing the monolayers again with PBS, infected cells were lysed with $0.5 \%$ Triton X-100 and the recovered bacteria were titered on TSB Congo Red plates after incubation at $37^{\circ} \mathrm{C}$. Percent invasion was determined by calculating the total recovered gentamicin-resistant colony forming unit per well after lysis divided by the total number of input bacteria per well.

Invasion $\%=($ total recovered bacteria $) /($ total input bacteria $) \times 100$

\section{Plaque assay}

Assays were performed as previously described. ${ }^{18}$ In brief, bacteria were grown overnight, subcultured into TSB and grown with agitation until reaching mid-log phase. Strains were standardized to an OD at $600 \mathrm{~nm}$ of 0.35 , washed in PBS and diluted in DMEM. The input bacteria were titered on TSB Congo Red plates before applying to six-well plates seeded to confluence with L2 cells. The six-well plates were then rocked at $37^{\circ} \mathrm{C}$ under $5 \% \mathrm{CO}_{2}$ for $2 \mathrm{~h}$. An agarose solution consisting of DMEM, $10 \%$ fetal bovine serum, $50 \mu \mathrm{g} \mathrm{ml}^{-1}$ gentamicin, $0.5 \%$ agarose (ISC Bioexpress; Kaysville, UT, USA) and compound was then applied to the monolayers. The plates were incubated at $37^{\circ} \mathrm{C}$ under $5 \% \mathrm{CO}_{2}$ for 3 days and then stained with $0.5 \%$ Neutral Red (Sigma-Aldrich) to visualize plaques. Efficiency of plaque formation was calculated by dividing the total number of plaques observed after staining by the total number of input bacteria. The average plaque size was determined by measuring 30 plaques, which were overall round in shape with irregular margins of clearing in the presence of compound and were perfectly circular when compound was not present.

Plaque efficiency $(\%)=($ total number of plaques $) /($ total input bacteria $) \times 100$

\section{RESULTS}

\section{Hit identification}

A summary of the hit identification process is shown in Figure 2. The 100000 compounds tested in the primary screen (in duplicate) produced 640 compounds that were considered active based on primary selection criteria $(0.64 \%$ hit rate). Of the 640 active compounds, 592 met selection criterion A, 39 met selection criterion $\mathrm{C}$ and 9 met selection criterion $\mathrm{B}$. The average $Z^{\prime}$ factor per plate tested in the primary screen was equal to $0.66\left(Z^{\prime}=1-\left(\left(3 \sigma_{\mathrm{c}+}\right.\right.\right.$ $\left.\left.\left.+3 \sigma_{\mathrm{c}-}\right)\right) /\left(\left|\mu_{\mathrm{c}+}-\mu_{\mathrm{c}-}\right|\right)\right)$, where $\sigma_{\mathrm{c}+}$ and $\sigma_{\mathrm{c}-}$ are the s.d. of the positive and negative controls and $\mu_{\mathrm{c}+}$ and $\mu_{\mathrm{c}-}$ are the means of the positive and negative controls, respectively).

The 640 active compounds were next tested in a dose-response study (in duplicate) monitoring both bacterial growth inhibition (OD) and VirF inhibition (CPRG hydrolysis). Of the 640 active 
$\sim 100,000$ small molecule library screened

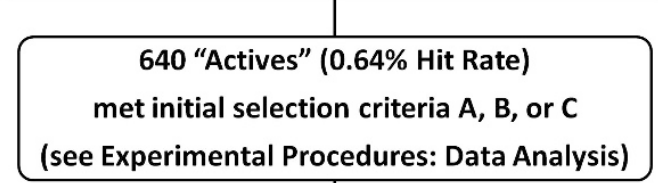

16 compounds passed all counter screens

- No $\beta$-galactosidase inhibition

- No bacterial growth inhibition

- IC $\mathrm{C}_{50} \leq 100 \mu \mathrm{M}$ for VirF inhibition

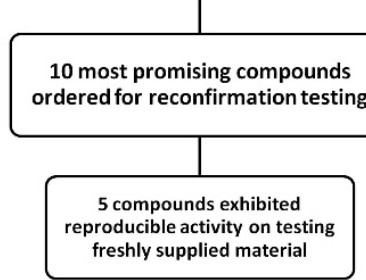

Figure 2 Flow chart depicting hit identification process for high-throughput screening (HTS).

compounds, only 52 inhibited CPRG hydrolysis while not inhibiting bacterial growth over the range of concentrations tested (for example, $\left.\mathrm{MIC}_{50}>100 \mu \mathrm{M}\right)$. These 52 compounds were further tested for direct $\beta$-galactosidase inhibition and 17 of the 52 compounds inhibited $\beta$-galactosidase in a dose-dependent manner. Therefore, only 35 of 640 active compounds appeared to inhibit the activity of VirF. Of the 35 compounds, only 16 had an $\mathrm{IC}_{50} \leqslant 100 \mu \mathrm{M}$ for VirF inhibition. On the basis of perceived novelty of scaffold (scaffolds that commonly found are less desirable as they are likely to have off-target effects), availability and potential toxicity concerns, we eliminated six of the compounds from further study. Fresh samples of the remaining 10 compounds were ordered from commercial vendors (seven from ChemDiv, three from Vitas-M Laboratory) for the reconfirmation study (below). The structures of these 10 compounds are shown in Table 1.

\section{Reconfirmation}

Each of the 10 compounds was tested in a modified 96-well plate version of the screening assay. Table 2 shows the results from the modified dose-response study. Out of the 10 compounds tested, only 5 reconfirmed. The $\mathrm{IC}_{50}$ values of these compounds ranged from 14 to $66 \mu \mathrm{M}$. Figure 3 shows the dose-response curves for the two most potent compounds, 19615 and 144092. The dose-response curves for the rest of the compounds can be found in Supplementary Figure 1.

\section{Identifying the highest concentration of compound not affecting} host cell or bacteria survival

As shown in Figure 4 and Supplementary Figure 2, the highest concentrations of compounds identified as having no observable effect on host cell or bacterial survival were used for virulence assays, and are as follows: $144092(6.25 \mu \mathrm{M}), 144143(6.25 \mu \mathrm{M}), 19615$ $(6.25 \mu \mathrm{M}), 153578(12.5 \mu \mathrm{M})$ and $24904(50 \mu \mathrm{M})$.

\section{Invasion assays}

Invasion assays were performed to identify compounds' effects on bacterial invasion of HeLa cells by having compounds present during
Table 1 Hits from $100 \mathrm{~K}$ ChemDiv screen that were rescreened from fresh samples

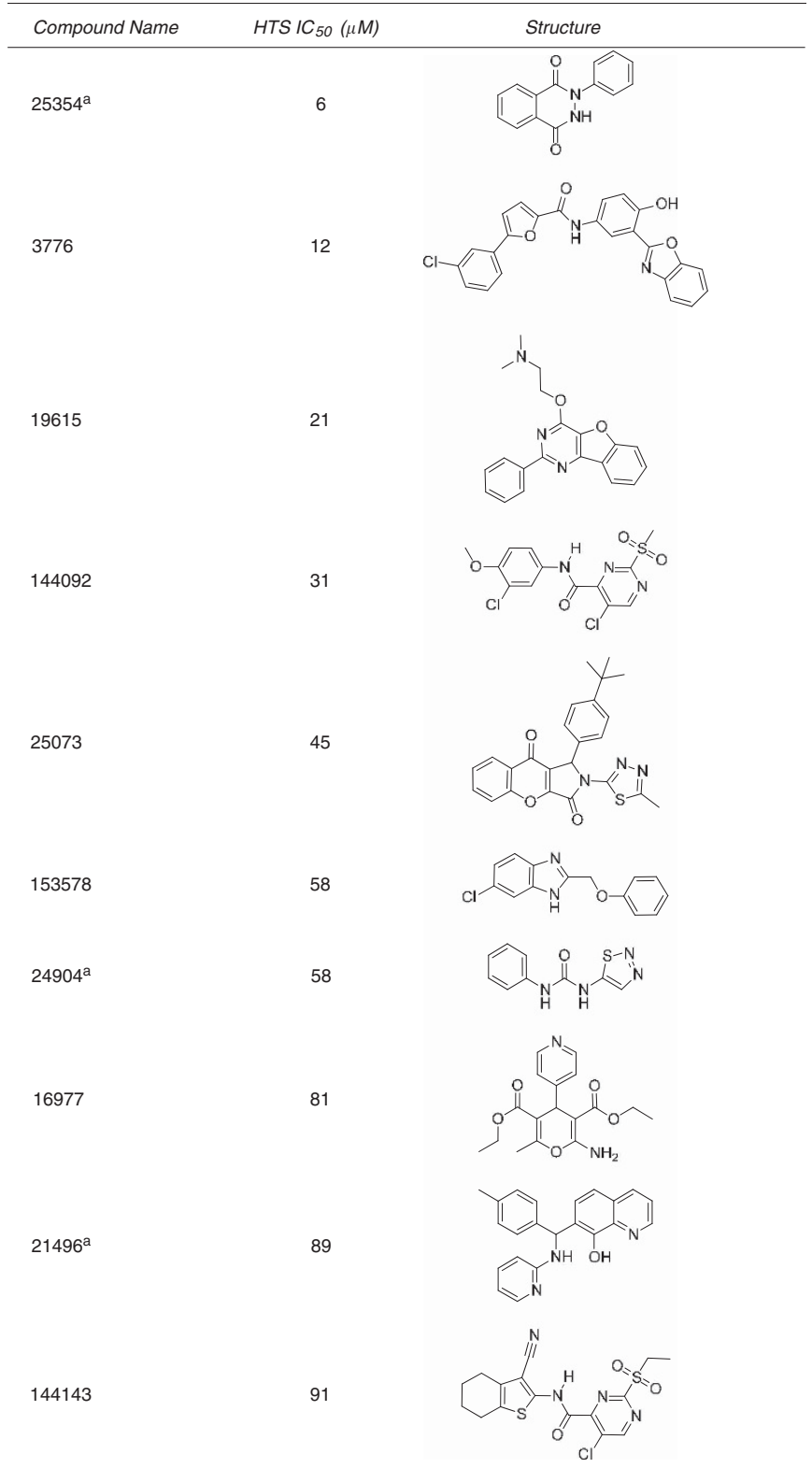

These compounds were also hits in original 42000 small molecule screen previously reported (Hurt et al. ${ }^{13}$ ).

bacterial subculture and invasion period or invasion period alone. As Figure 5 shows, when present during bacterial subculture and invasion period, 144092 was the only compound that lowered invasion efficiency compared with wild-type S. flexneri 2457T $(0.14 \%$ and $0.30 \%$ respectively); however, it displayed no effect on invasion when present during invasion period alone $(0.30 \%)$.

The remainder of the compounds displayed hyperinvasion when compared with wild-type invasive bacteria (compounds 144143 and 19615, 2.1-fold increase; compound 153578, 9.6-fold increase; compound 24904, 9.8-fold increase). As a control, we tested the virulence plasmid-cured derivative of $S$. flexneri $2457 \mathrm{~T}, \mathrm{BS} 103$, in the invasion assay in the presence of compounds. The results show no BS103 surviving gentamicin treatment (data not shown). 
Table 2 Reconfirmation: compound $\mathrm{IC}_{50}$ values and toxicities from fresh samples ${ }^{a}$

\begin{tabular}{llccl}
\hline Compound & Source & $\begin{array}{c}I_{50} \\
(\mu \mathrm{m})^{\mathrm{b}}\end{array}$ & $\begin{array}{c}\text { Max Immunocytotoxic concen- } \\
\text { tration }(\mu \mathrm{m})^{\mathrm{c}}\end{array}$ & $\begin{array}{l}\text { Bacterial growth } \\
\text { inhibition }^{\mathrm{d}}\end{array}$ \\
\hline 19615 & Vitas-M & 14 & 6.25 & $2 \% @ 100 \mu \mathrm{m}$ \\
144092 & ChemDiv & 23 & 6.25 & $13 \% @ 100 \mu \mathrm{M}$ \\
144143 & ChemDiv & 23 & 6.25 & $14 \% @ 100 \mu \mathrm{m}$ \\
153578 & ChemDiv & 37 & 12.5 & $19 \% @ 100 \mu \mathrm{m}$ \\
24904 & ChemDiv & 66 & 50 & $13 \% @ 50 \mu \mathrm{m}$
\end{tabular}

${ }^{a}$ Compounds 25354, 3776, 25073, 16977 and 21496 did not reconfirm and were not tested for toxicity.

Log $\left(\mathrm{IC}_{50}\right)$ values and their associated errors can be found in Supplementary Table 1 . The estimated percent errors for each reconfirmed compound were $\leqslant 2 \%$.

cMaximum noncytotoxic concentrations were determined as the highest concentrations at which there were no observable morphological effects on the cells.

${ }^{\mathrm{d}}$ The $\%$ inhibition of bacterial growth values are averages of three determinations with s.e. of $3 \%$.

\section{Efficiency of plaque formation}

Plaque assays were performed to identify potential effects of compounds on cell-to-cell spread using L2 cells, with compound present only in the agarose overlay. Overall, three compounds caused significant reductions in the efficiency of plaque formation for wild-type S. flexneri $2457 \mathrm{~T}$ relative to the no compound control (144092: 23\%, 19615: 19\% and 153578: 24\% efficiency), whereas 144143 had no effect on the efficiency of plaque formation; see Figure 6 (Monolayers with $50 \mu \mathrm{m} 24904$ did not survive the duration of the assay). The average plaque size for 144092 was reduced to $81 \%$ of wild-type plaque sizes ( 0.87 versus $1.08 \mathrm{~mm}$ ), whereas plaque sizes were unaffected by the other compounds; see Figure 7 .

\section{DISCUSSION}

S. flexneri is a Gram-negative enteropathogen that infects the colonic epithelium of its host. Current treatments for shigellosis include fluoroquinolones, such as ciprofloxacin, and cephalosporins. ${ }^{19}$ Along with a rise in antibiotic resistance, ${ }^{20-22}$ another problem with the current treatment options is that they also harm the normal colonic microbiota of the host. Upsetting the colonic microbiota can lead to digestion problems as well as secondary infections, such as by Clostridium difficile, ${ }^{23}$ which can be more severe than the original infection. In theory, a virulence therapy would bypass these issues as its target would not be present in nonpathogenic bacteria; therefore, it would not harm the normal colonic microbiota. A common criticism against the pursuit of virulence-targeted therapies is that as they are not bactericidal, they do not directly clear an infection. However, by inhibiting VirF, the main transcriptional activator of virulence genes in S. flexneri, host cell invasion and cell-to-cell spread (both of which are required to maintain the infection) will be attenuated. As shown in Figure 1, not only does VirF activate virulence genes controlling invasion and spread, but it also activates virulence genes necessary for macrophage escape. If macrophage escape can be prevented, then the infection should be more rapidly cleared by the host's immune system. If this theory holds, then VirF should be an effective target for a virulence-directed therapy against shigellosis.

To identify an inhibitor of VirF, we carried out a high-throughput screen (HTS) of 100000 small molecules using our previously described $^{13}$ bacteria-based, VirF-driven, $\beta$-galactosidase reporter assay. The primary HTS identified 640 small molecules that met initial selection criteria for VirF inhibition; see Figure 2. The 640 small molecules were tested in a series of counter screens to eliminate any false positives that inhibited bacterial growth or $\beta$-galactosidase
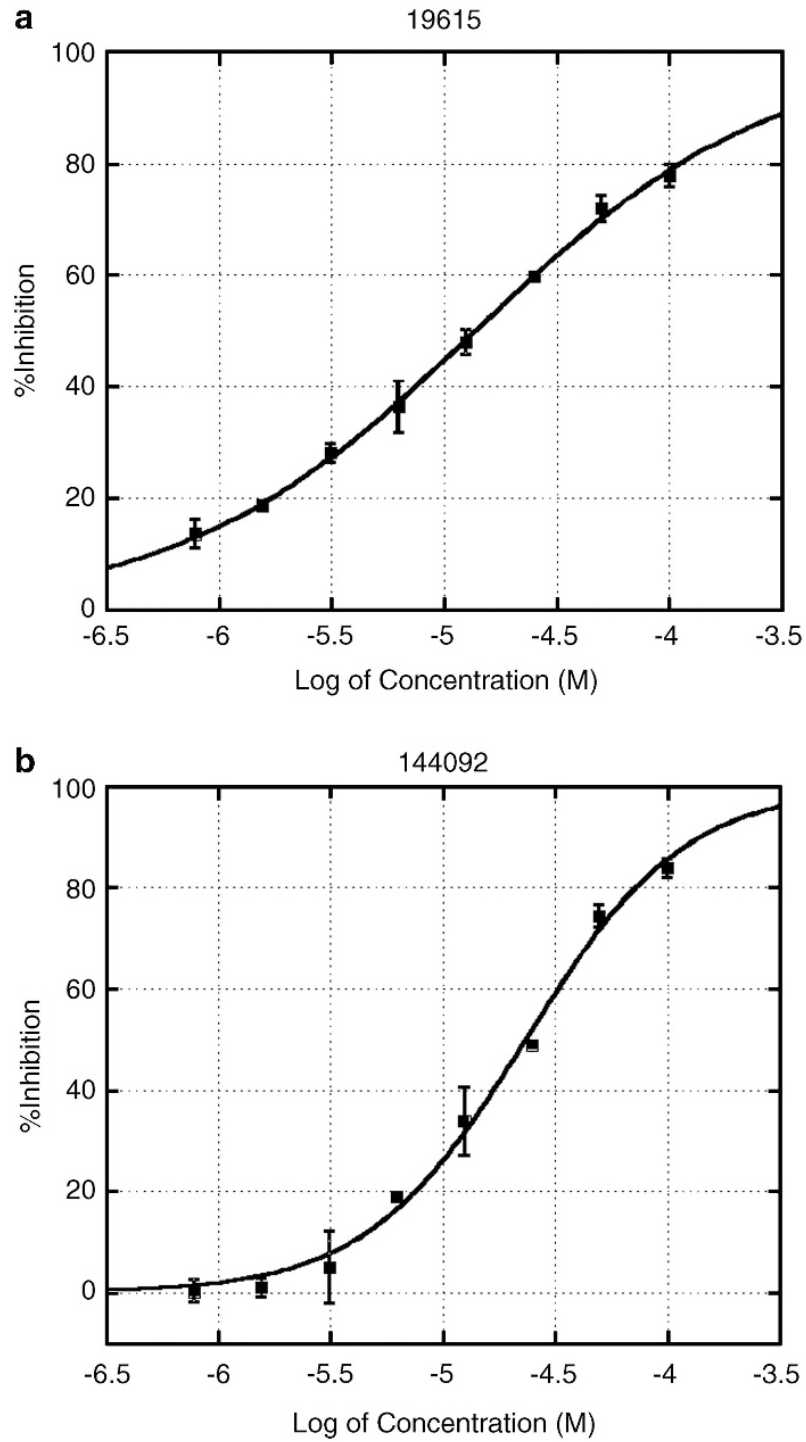

Figure 3 Representative dose-response curves from reconfirmation study. All concentrations were tested in triplicate. The average $\%$ inhibition for each concentration is represented on the plots as solid black squares. Data were normalized for bacteria growth and background signal (positive control average: simulates complete VirF inhibition) was removed from data before plotting. Curves were fit to the equation listed in Materials and methods, and represented on the plots as solid black lines. For the fits, $M 1=\log \left(I_{50}\right)$ and $M 2=$ hill slope. (a) Dose-response curve for compound 19615. Concentrations ranging from 100 to $0.78 \mu \mathrm{m}$ were tested. (b) Dose-response curve for compound 144092. Concentrations ranging from 100 to $0.78 \mu \mathrm{m}$ were tested.

directly and to verify that the compounds exhibited dose-dependent activity. A total of 16 compounds passed all selection criteria and counter screens. On the basis of the novelty of scaffold, availability and potential toxicity concerns, we eliminated six of the compounds from further study. The remaining 10 compounds were ordered from commercial sources and tested in a dose-response study. The structures and initial $\mathrm{IC}_{50}$ values of the 10 compounds are shown in Table 1 . As Table 2 shows, only 5 of the 10 compounds reconfirmed when fresh, pure samples were tested. Mass spectroscopy verified that the new samples of the five compounds that did not reconfirm were of the appropriate mass (data not shown), 

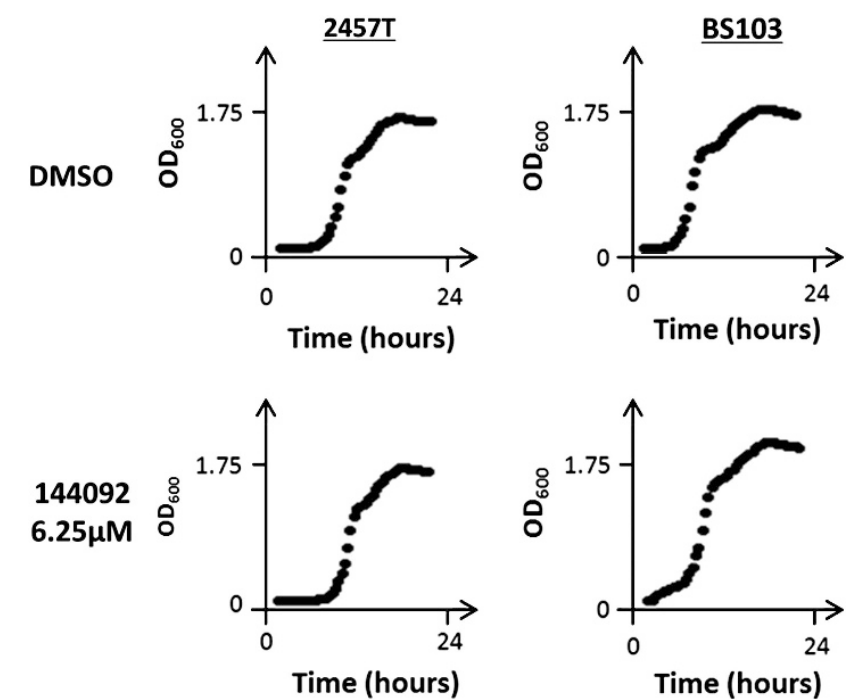

Figure 4 Bacterial toxicity of dimethyl sulfoxide (DMSO) control and 144092. Dilutions of compounds were added to bacterial cultures and $\mathrm{OD}_{600}$ was monitored for $24 \mathrm{~h}$ and plotted over time. Shown are the growth curves for the 1\% DMSO control and compound 144092 at $6.25 \mu \mathrm{m}$, the highest tested concentration that showed no growth inhibition. (See Supplementary Figure 2 for other compounds.)

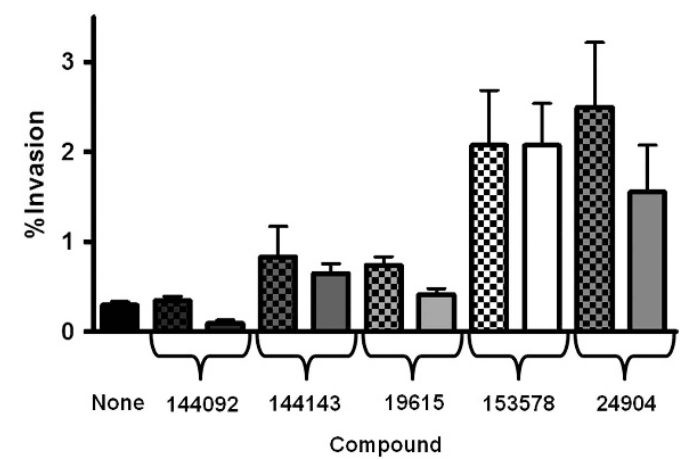

Figure 5 Invasion assay with S. flexneri 2457T. Graph depicts \% invasion of S. flexneri 2457T into monolayers of HeLa cells in the presence of compounds as described in Materials and Methods. Each compound was dosed at the highest concentration that had no effect on cell or bacterial survival as identified in Figure 3. Compounds were given during the invasion period only (checkered bar graphs) and during the exponential phase of bacterial growth and remained present during the invasion period (solid bar graphs).

suggesting that the fresh samples were the correct compounds. Low reconfirmation rates are not uncommon in HTS follow-up studies. $^{24}$ A variety of factors including library compound degradation, library compound impurities and compound transfer errors can increase the number of false-positive results obtained from an HTS. The most likely explanation for our low reconfirmation rate is that the active compounds from the HTS library are degradation products of the original compounds. The HTS library is stored in dimethyl sulfoxide and has been subjected to multiple freeze-thaw cycles, which increases the likelihood of compound degradation. Owing to the difficulty and unpredictable nature of determining the active degradation products from the HTS library, we decided to

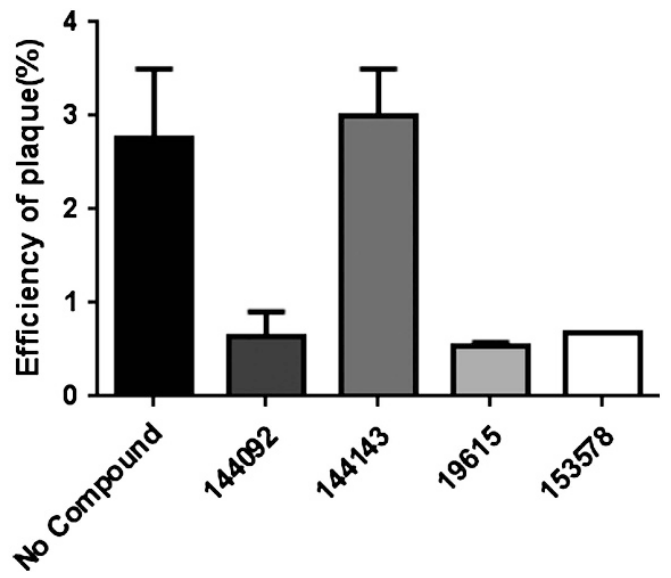

Figure 6 Efficiency of plaque formation with S. flexneri 2457T. Graph depicts \% efficiency of plaque formation of $S$. flexneri $2457 T$ in the presence of compounds after initial infection into monolayers of $L 2$ cells as described in Materials and Methods. Each compound was dosed at the highest concentration that had no effect on cell or bacterial survival as identified in Figure 3. Compound 24904 is not depicted on graph because of its toxicity toward the L2 monolayers over the duration of the assay.

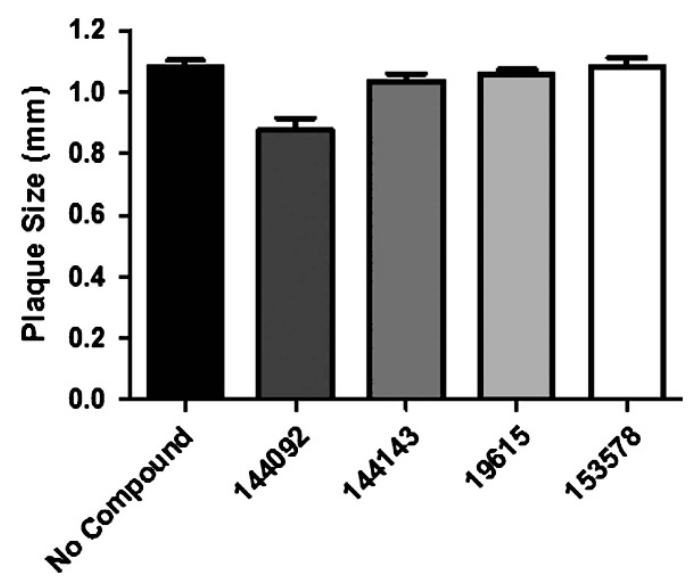

Figure 7 Size of plaques from plaque formation assay with $S$. flexneri 2457T. The graph displays average plaque size of $S$. flexneri 2457T in the presence of compounds in L2 cells and visualized by Neutral Red staining as described in Materials and Methods. The concentration of each compound used in the assay was the highest concentration that demonstrated no effect on host or bacterial survival as identified in Figure 3. No compound was used as a control for wild-type S. flexneri 2457T plaque sizes.

move forward with only the five compounds that reconfirmed from new, clean samples.

The reconfirmed compounds were considered promising for a variety of reasons. All of the compounds had $\mathrm{IC}_{50}$ values for VirF inhibition $<100 \mu \mathrm{M}$, with compound 19615 being the most potent $\left(\mathrm{IC}_{50}=14 \mu \mathrm{M}\right)$. As these $\mathrm{IC}_{50}$ values were determined using an Shigella-based reporter system, we know each compound can cross through the bacterial membrane and bind its target. Compounds 144092 and 144143 are close analogus and share a core pyrimidine scaffold, which makes them attractive candidates for future optimization via structure activity relationship studies. Compound 153578 has a benzimidazole core that is similar to the benzimidazole compounds 
that were shown to inhibit the virulence regulator of $Y$. pseudotuberculosis, LcrF. ${ }^{9,11,12}$ Interestingly, LcrF and VirF are both members of the same class of transcriptional activators, the AraC family. Compound 24904 is a known plant defoliant, thidiazuron, which is reported to have low acute toxicity toward humans. ${ }^{25}$

The compounds were next evaluated in HeLa cell monolayer invasion and L2 cell monolayer plaque assays as models for the infection process. As shown in Figure 5, one compound, 144092, was able to reduce the \% invasion relative to the no compound control when the compound was administered during the exponential growth phase of the bacterial subculture in addition to being present through the invasion period. The compound had no effect on \% invasion when it was given at the time of invasion only. The lack of effect in the latter case is most likely due to VirF activating virulence genes during exponential growth in the absence of the compound. Those virulence proteins would then be already present when the compound was added during invasion; hence, the bacteria would still be able to invade the host cells. The ineffectiveness of the other four compounds was not unexpected because all of the compounds were administered at concentrations below their IC $_{50}$ values because of toxicity toward the cell monolayers at higher concentrations (see Table 2 and Supplementary Table 2). However, it was unexpected that the other four compounds displayed 'hyperinvasion' relative to no compound controls. Control assays were performed to verify that compounds were not artificially raising $\%$ invasion by protecting the bacteria from gentamicin. It is possible that the compounds could be altering the HeLa cell monolayer in a nonobservable manner making it more prone to bacterial infection. Further studies will need to be performed to determine the cause of the 'hyperinvasion'.

Three of the compounds 144092, 19615 and 153578 lowered the efficiency of plaque formation by $S$. flexneri $2457 \mathrm{~T}$, indicating that the compounds attenuated the cell-to-cell spread of the bacteria (see Figure 6). This result was surprising as only compound 144092 lowered the invasion efficiency of S. flexneri $2457 \mathrm{~T}$ during the invasion assays. However, only 144092 had any effect, albeit quite modest, on plaque size. Plaque size is dependent upon a number of factors including cell-to-cell spread and bacterial growth. Also, the plaque size is assessed after 3 days. The stability of the compounds over that period is not known, so it is hard to interpret effects on plaque size without further studies.

The invasion and plaque formation efficiency results suggest that the compounds may be exhibiting differential effects on VirF activity dependent upon which promoter VirF is binding. As shown in Figure 1, VirF can bind to both the virB and icsA (also called $\operatorname{vir} G$ ) promoters. Activation of the virB promoter leads to the transcription of virulence genes associated with macrophage escape and gastrointestinal cell invasion, whereas activation of the icsA promoter leads to the transcription of $i c s A$, which is associated only with the cell-tocell spread of the bacteria (for example, actin polymerization leading to motility). It is possible that compounds 19615 and 153578 could have a stronger inhibitory effect on VirF binding to (and hence activation of) the icsA promoter than the $\operatorname{vir} B$ promoter; this would explain the contradictory results seen between the two assays. As the reporter assay used for the HTS only monitored VirF activating the virB promoter, we are currently developing assays that directly measure VirF binding to each of its promoters ( $\operatorname{vir} B$ and $i c s A$ ). Another possibility that we are investigating is that these compounds could be directly inhibiting the actin polymerase activity associated with IcsA.

It is noteworthy that these compounds (144092, 19615 and 153578), directly from our HTS, have not yet been optimized in any way and were tested well below their $\mathrm{IC}_{50}$ values for VirF inhibition (due to cytotoxicity). The fact that the compounds produced even modest attenuation of the infection process at the concentrations tested is intriguing. Compound 144092 had a significant effect on invasion at a concentration of about $25 \%$ of its $\mathrm{IC}_{50}$ in the VirF screen. Our VirF screening assays involve overnight incubations, whereas the invasion assays are only incubated for a total of 4-6h. The longer time frame of the screening assays may allow small amounts of active VirF to generate significant quantities of $\beta$-galactosidase, resulting in a higher $\mathrm{IC}_{50}$. In vivo there is an amplification factor as VirF activates VirB, which in turn activates the invasion proteins. Inhibition of VirF could result in a much greater reduction in the invasion proteins due to this amplification, which is not present in our screening plasmid. The Shigella protein $\mathrm{H}-\mathrm{NS}$ binds to both $v i r B$ and $i c s A$ promoter regions and represses their transcription. It may be that our artificial screening plasmid may be less susceptible to H-NS repression than the natural, $200 \mathrm{~Kb}$ virulence plasmid, making it harder to block VirF activation of gene expression.

Compound 144092 is the most promising candidate for hit-to-lead development as it was able to reduce initial invasion efficiency $(53 \%$ reduction), plaque efficiency $(77 \%$ reduction) and plaque size (Figure $7,19 \%$ reduction) at a concentration $(6.25 \mu \mathrm{M})$ that had no effect on bacterial or host cell viability. Furthermore, 144092 and 144143 share the same pyrimidine core. The differential activity of these analogs provides key insights into which regions of compound 144092 should be focused upon for analog development. It is reasonable to expect that with further optimization of the chemical structures, an increase in efficacy in both the invasion and plaque efficiency assays as well as a decrease in cytotoxicity will be achieved.

In this report, we have identified five compounds with VirF inhibitory properties from a 100000 compound high-throughput screen. We have validated the approach to our HTS by providing evidence that it is possible to attenuate the virulence of $S$. flexneri by inhibiting VirF with a small molecule. We have identified three small molecules that were capable of blocking the cell-to-cell spread of S. flexneri at concentrations that do not affect bacterial or cell growth. These results also indicate that the compounds are able to penetrate the bacteria and the host cells, an attribute that is very encouraging for further development. As all three of these compounds were given after initial bacterial invasion in the plaque assay, we have also validated the potential of a VirF inhibitor for treating an active infection (a common criticism of virulence therapies). Furthermore, one of the three compounds was able to lower the initial invasion efficiency of S. flexneri. This compound, 144092, is a promising chemical scaffold for hit-to-lead development as an anti-virulencetargeting therapeutic for the treatment of shigellosis.

\section{ACKNOWLEDGEMENTS}

We gratefully acknowledge Martha J Larsen and Tom McQuade at Center of Chemical Genomics, Life Sciences Institute, University of Michigan, for highthroughput screening automation and sample management. This research was supported by the National Institutes of Health (AI85179, GAG; AI24656, ATM; and T32-GM007767, AAE trainee) and the American Foundation for Pharmaceutical Education (AAE, scholarship).

\section{DISCLAIMER}

After submission of this manuscript, a paper by Koppolu et al. was published in the November issue of Infection and Immunity that describes the testing of a small molecule inhibitor of AraC-family transcriptional activators against the $S$. VirF. ${ }^{26}$ Their results confirm our results reported here that inhibition of VirF results in reduction of invasion and requires preincubation of $S$. with the inhibitor. 
1 Kumarasamy, K. K. et al. Emergence of a new antibiotic resistance mechanism in India, Pakistan, and the UK: a molecular, biological, and epidemiological study. Lancet Infect. Dis. 10, 597-602 (2010).

2 Lewis, K. Platforms for antibiotic discovery. Nat. Rev. Drug Discov. 12, 371-387 (2013).

3 Jenssen, W. D., Thakker-Varia, S., Dubin, D. T. \& Weinstein, M. P. Prevalence of macrolides-lincosamides-streptogramin $B$ resistance and erm gene classes among clinical strains of Staphylococci and Streptococci. Antimicrob. Agents Chemother. 31, 883-888 (1987).

4 Marty, F. M. et al. Emergence of a clinical daptomycin-resistant Staphylococcus aureus isolate during treatment of methicillin-resistant Staphylococcus aureus bacteremia and osteomyelitis. J. Clin. Microbiol. 44, 595-597 (2006).

5 Petrella, S. et al. Genetic basis for natural and acquired resistance to the diarylquinoline R207910 in Mycobacteria. Antimicrob. Agents Chemother. 50, 2853-2856 (2006).

6 Tsiodras, S., Gold, H. \& Sakoulas, G. Linezolid resistance in a clinical isolate of Staphylococcus aureus. Lancet 358, 207-208 (2001).

7 Alksne, L. E. Virulence as a target for antimicrobial chemotherapy. Expert. Opin. Investig. Drugs 11, 1149-1159 (2002).

8 Setti, E. L. \& Micetich, R. G. New trends in antimicrobial development. Curr. Med. Chem. 5, 101-113 (1998).

9 Bowser, T. E. et al. Novel anti-infection agents: small-molecule inhibitors of bacterial transcription factors. Bioorg. Med. Chem. Lett. 17, 5652-5655 (2007).

10 Hung, D. T., Shakhnovich, E. A., Pierson, E., Mekalanos, J. J. \& Hung, D. T. Smallmolecule inhibitor of Vibrio cholerae virulence and intestinal colonization. Science 310, 670-674 (2005).

11 Garrity-Ryan, L. K. et al. Small molecule inhibitors of LcrF, a Yersinia pseudotuberculosis transcription factor, attenuate virulence and limit infection in a murine pneumonia model. Infect. Immun. 78, 4683-4690 (2010).

$12 \mathrm{Kim}, \mathrm{O}$. K. et al. N-hydroxybenzimidazole inhibitors of the transcription factor LcrF in Yersinia: novel antivirulence agents. J. Med. Chem. 52, 5626-5634 (2009).

13 Hurt, J. K., McQuade, T. J., Emanuele, A., Larsen, M. J. \& Garcia, G. A. Highthroughput screening of the virulence regulator VirF: a novel antibacterial target for Shigellosis. J. Biomol. Screen. 15, 379-387 (2010).

14 Qi, M. S., Yoshikura, H. \& Watanabe, H. Virulence phenotypes of Shigella flexneri 2a virulent mutant 24570 can be complemented by the plasmid-coded positive regulator virF gene. FEMS Micrbiol. Lett. 71, 217-221 (1992).

15 Formal, S. B., Dammin, G. J., Labrec, E. H. \& Schneider, H. Experimental Shigella infections: characteristic of a fatal infection produced in guinea pigs. J. Bacteriol. 75 604-610 (1958)

16 Maurelli, A. T., Blackmon, B. \& Curtiss, R. III Loss of pigmentation in Shigella flexner $2 \mathrm{a}$ is correlated with loss of virulence and virulence associated plasmid. Infect. Immun. 43, 397-401 (1984).

17 Hale, T. L. \& Formal, S. B. Protein synthesis in HeLa or Henle 407 cells infected with Shigella dysenteriae 1, Shigella flexneri 2a, or Salmonella typhimurium W118. Infect. Immun. 32, 137-144 (1981).
18 Oaks, E. V., Hale, T. L. \& Formal, S. B. Plaque formation by virulent Shigella flexneri. Infect. Immun. 48, 124-129 (1985).

19 World Health Organization. Guidelines for the Control of Shigellosis, Including Epidemics Due to Shigella Dysenteriae Type 1 (WHO Press, World Health Organization, Geneva, Switzerland, 2005).

20 CDC. Outbreaks of multidrug-resistant Shigella sonnei gastroenteritis associated with day care centers-Kansas, Kentucky, and Missouri, 2005. MMWR Morb. Mortal. Wkly. Rep. 55, 1068-1071 (2006).

21 Qiu, S. et al. Emergence of resistance to fluoroquinolones and third-generation cephalosporins in Shigella flexneri subserotype 1c isolates from China. Clin. Microbiol Infect. 18, E95-E98 (2012).

22 Replogle, M. L., Fleming, D. W. \& Cieslak, P. R. Emergence of antimicrobial-resistant shigellosis in Oregon. Clin. Infect. Dis. 30, 515-519 (2000).

23 Bignardi, G. E. Risk factors for Clostridium difficile infection. J. Hosp. Infect. 40, 1-15 (1998).

24 Zhang, J., Chung, T. \& Oldenburg, K. Confirmation of primary active subtances from high throughput screening of chemical and biological populations: a statistical approach and practical considerations. J. Comb. Chem. 2, 258-265 (2000).

25 U.S. Environmental Protection Agency. Reregistration Eligibility Decision (RED) Fact Sheet for Thidiazuron, EPA-738-F-04-012 (US EPA, Washington, DC, 2005).

26 Koppolu, V. et al. Small-molecule Inhibitor of the Shigella flexneri master virulence regulator VirF. Infect. Immun. 81, 4220-4231 (2013).

27 Tobe, T., Yoshikawa, M., Mizuno, T. \& Sasakawa, C. Transcriptional control of the invasion regulatory gene virB of Shigella flexneri: activation by virF and repression by H-NS. J. Bacteriol. 175, 6142-6149 (1993).

28 Tobe, T., Nagai, S., Okada, N., Adler, B., Yoshikawa, M. \& Sasakawa, C. Temperatureregulated expression of invasion genes in Shigella flexneri is controlled through the transcriptional activation of the virB gene on the large plasmid. Mol. Microbiol. 5, 887-893 (1991).

29 Hilbi, H. et al. Shigella-induced apoptosis is dependent on caspase-1 which binds to IpaB. J. Biol. Chem. 273, 32895-32900 (1998).

30 Blocker, A. et al. The Tripartite type III secretion of Shigella flexneri inserts IpaB and IpaC into host membranes. J. Cell. Biol. 147, 683-693 (1999).

31 Espina, M. et al. IpaD localizes to the tip of the type III secretion system needle of Shigella flexneri. Infect. Immun. 74, 4391-4400 (2006).

32 Schroeder, G. N. \& Hilbi, H. Molecular pathogenesis of Shigella spp.: controlling host cell signaling, invasion, and death by type III secretion. Clin. Microbiol. Rev. 21, 134-156 (2008)

33 Tran, C. N. et al. A multifactor regulatory circuit involving H-NS, VirF and an antisense RNA modulates transcription of the virulence gene icsA of Shigella flexneri. Nucleic Acids Res. 39, 8122-8134 (2011).

34 Bernardini, M. L., Mounier, J., d'Hauteville, H., Coquis-Rondon, M. \& Sansonetti, P. J. Identification of icsA, a plasmid locus of Shigella flexneri that governs bacterial intraand intercellular spread through interaction with F-actin. Proc. Natl Acad. Sci. USA 86, 3867-3871 (1989).

Supplementary Information accompanies the paper on The Journal of Antibiotics website (http://www.nature.com/ja) 